\title{
Clinical use of antimicrobial regional limb perfusion in horses: 174 cases (1999-2009)
}

\author{
Luis M. Rubio-Martínez, DVM, PhD, DACvs; Colette R. Elmas, DVM; \\ Belinda Black, BVMs; Gabrielle Monteith, BS
}

\begin{abstract}
Objective-To describe the clinical use of regional limb perfusion with antimicrobials (A-RLP), complications, and outcome in a large series of patients.

Design-Retrospective case series.

Animals-174 horses.

Procedures-Medical records of horses treated with A-RLP between 1999 and 2009 were reviewed. Signalment, primary complaint, horse use, etiology, duration of clinical signs, previous treatment, structures involved, concurrent conditions, A-RLP characteristics, additional treatments, complications, and outcome were recorded. At long-term follow-up, 2 outcomes were investigated: survival rate and return to previous use at the same or higher level. Univariate and multivariate logistic regression analyses were conducted.

Results-Group 1 (96 horses) included septic synovitis. Group 2 (50 horses) included extrasynovial lacerations (23 horses) and fresh, minimally contaminated intrasynovial lacerations without evidence of established synovial infection (27 horses). Group 3 (28 horses) included miscellaneous other conditions. Only minor complications were reported in $12.26 \%$ of horses that received IV $(n=155)$ and $33 \%$ of horses that received intraosseous (27) A-RLP. Horses with septic synovitis had a lower survival rate $(53.43 \%)$ than did horses with lacerations $(91.89 \%)$. Within group 2, no significant differences in short- or long-term outcomes were found between horses with extrasynovial and fresh, minimally contaminated intrasynovial lacerations. For the horses returning to previous use, $80 \%$ of horses with septic synovitis and $72.72 \%$ of horses with lacerations were performing at the same or higher level at the time of follow-up.

Conclusions and Clinical Relevance-The results of the present study indicated that A-RLP is a safe technique with minimal adverse effects. The IV route presented fewer complications than intraosseous injection. Horses with infection of synovial structures had a lower survival rate than did those with acute, minimally contaminated intrasynovial lacerations. The latter had a similar prognosis for horses with extrasynovial lacerations treated with A-RLP. (J Am Vet Med Assoc 2012;241:1650-1658)
\end{abstract}

$S_{a}$ ystemic and locoregional antimicrobials are often administered in combination for the treatment of orthopedic infections in horses. Regional limb perfusion with antimicrobials has become a common practice for treatment of infections in the distal aspect of the limb of horses. ${ }^{1-5}$ The A-RLP technique consists of the administration of an antimicrobial solution into the vasculature of a selected portion of the limb that has been isolated from the systemic circulation by the controlled application of a tourniquet. ${ }^{2}$ After the tourniquet is applied, the antimicrobial solution is injected into the isolated portion of the vascular system. Both the IV and intraosseous routes can be used with similar results. ${ }^{6-8}$ The antimicrobial concentrations

From the Department of Clinical Studies, Ontario Veterinary College, University of Guelph, Guelph, ON NIG 2W1, Canada. Dr. RubioMartínez's present address is Department of Companion Animal Studies, Faculty of Veterinary Science, University of Pretoria, 0110 Onderstepoort, South Africa.

Presented in part at the Meeting of the European College of Veterinary Surgeons, Helsinki, July 2010, and at the Congress of the World Equine Veterinary Association, Hyderabad, India, November 2011.

Address correspondence to Dr. Rubio-Martínez (luis.rubiomartinez@ hotmail.com).

\begin{tabular}{ll}
\hline & \multicolumn{1}{c}{ ABBREVIATIONS } \\
A-RLP & Regional limb perfusion with antimicrobials \\
CI & Confidence interval \\
ISCL & Intrasynovial continuous lavage \\
RLP & Regional limb perfusion \\
\hline
\end{tabular}

achieved in synovial fluid and bone tissue after A-RLP are much higher than those after systemic administration, ${ }^{7,9-11}$ and experimental infections have responded favorably to A-RLP. ${ }^{12}$ Reported complications of A-RLP include thrombophlebitis of the injected vein, soft tissue inflammation over the intraosseous infusion site, difficult injection into the medullary cavity of the bone, and osteonecrosis of the injected bone. ${ }^{3,6,13} \mathrm{On}$ the basis of clinical experiences and anecdotal clinical reports, ${ }^{1,5,11,12,14}$ it is believed that the use of A-RLP has helped increase the successful outcome of horses with orthopedic infections. However, information about ease of use, complications, and short- and long-term results after clinical application of A-RLP in a large number of cases is lacking. The objective of the study reported here was to describe the clinical use of A-RLP and its complications and outcome in a large series of horses. 


\section{Materials and Methods}

Case selection criteria-Medical records from horses hospitalized at the Ontario Veterinary College Teaching Hospital between October 1999 and May 2009 that received A-RLP were reviewed.

Medical records review-Retrieved data included signalment (breed, sex, and age), use, duration of clinical signs, treatment prior to admission, structures involved, concurrent conditions, A-RLP characteristics, additional medical or surgical treatments, and hospitalization time. Characteristics of the A-RLP included total number of A-RLPs performed, starting day, time interval between A-RLP procedures, route of administration (IV or intraosseous), administration site, whether performed under general anesthesia or standing sedation, antimicrobial used, antimicrobial dose, total perfusate volume, use of local anesthetic in the perfusate (amount of local anesthetic used), use of perineural anesthetic block proximal to the tourniquet, and duration of tourniquet placement. Recorded technical and clinical complications associated with the perfusions were also retrieved. Complications were considered minor as long as these had no consequences for the well-being of the patient or did not require a major change in the treatment plan. Minor complications included mild phlebitis, mild transient localized swelling, difficult injection, or screw breakage. Complications were considered major when important consequences to the patient were observed (ie, severe thrombophlebitis, tissue sloughing, complication requiring a change in treatment plan, or bone fracture at the injection site). Patients with incomplete records were excluded.

Horses included in the study were divided into 3 groups according to the main clinical sign: septic synovitis (group 1), lacerations (group 2), or any other clinical condition (group 3). Horses with an infected synovial structure (joint, synovial sheath, or bursa) were included in group 1. A diagnosis of septic synovial structure was confirmed if organisms were seen on cytologic examination of synovial fluid or if results of bacterial culture of synovial fluid or a membrane sample were positive. Additionally, a diagnosis of septic synovitis was made if there were obvious signs of contamination in chronic synovial lacerations or the results of synovial fluid analysis met $\geq 3$ of the following criteria: WBC count $>30,000$ cells $/ \mu \mathrm{L}$ (reference range, 50 to 500 cells $/ \mu \mathrm{L}$ ), $>90 \%$ polymorphonuclear leukocytes (reference range, $\leq 10 \%$ ), total protein concentration $>4$ g/dL (reference range, 0.8 to $2.5 \mathrm{~g} / \mathrm{dL}$ ), and degenerative changes in polymorphonuclear leukocytes. ${ }^{15}$ Horses with fresh, minimally contaminated lacerations involving synovial structures did not meet criteria for synovial sepsis and were therefore included in group 2. Variables investigated in the synovial sepsis group (group 1) were breed, sex, age (both as a continuous and categorical variable [foal $\leq 6$ months of age vs adults]), use, duration of clinical signs ( $<24$ hours, 1 to 7 days, or $>7$ days), type of synovial structure affected (joint, sheath, or bursa), etiology of infection (hematogenous, traumatic, iatrogenic, or unknown), number of synovial structures affected per horse ( 1 vs $>1$ ), presence of osteomyelitis, other concurrent lesions, tendon involvement, number of A-RLPs performed, administration of systemic antimicrobials, intrasynovial local antimicrobials, use of arthroscopic or tenoscopic lavage, and use of ISCL with isotonic fluids. Group 2 included horses with lacerations not involving a synovial structure and horses that had lacerations involving a synovial structure but that did not meet the criteria for established infection. The latter typically included horses with fresh, minimally contaminated intrasynovial lacerations because these lacerations were considered to not have an established, ongoing septic process. In some of these cases, synovial fluid analysis revealed cytologic parameters within the reference ranges or well below those values indicated in group 1 . Results of cultures when available were negative. In some cases, a synovial fluid sample was not available as fluid had drained out through the laceration; however, contamination was minimal. Variables investigated in group 2 included breed, sex, and age (both as a continuous and categorical variable [foal $\leq 6$ months of age vs adults]), use, duration of clinical signs ( $<24$ hours, 1 to 7 days, or $>7$ days), synovial involvement (extrasynovial vs intrasynovial), type of synovial structure affected (joint, sheath, or bursa), number of synovial structures affected per horse ( 1 vs $>1$ ), bone involvement, tendon involvement, number of A-RLPs performed, administration of systemic antimicrobials prior to admission to the hospital, intrasynovial local antimicrobials, use of tenoscopic synovial lavage, and ISCL with isotonic fluids. Horses that received A-RLP for any other clinical condition were included in group 3.

Short-term outcome was determined at time of hospital discharge by evaluation of medical records to determine whether horses were successfully discharged from the hospital, euthanized for the primary complaint, or euthanized for an unrelated reason. Long-term outcome was determined at the time of follow-up ( $\geq 3$ months after discharge) by means of a telephone questionnaire administered to the owners or trainers. The purposes of the questionnaire were to determine whether horses were alive, had been euthanized because of the primary complaint, or had been euthanized for an unrelated reason and whether horses had returned to their previous use. During the questionnaire, the level of athletic performance that the horse had achieved prior to the occurrence of the condition under investigation was established, and then it was investigated whether, in the owner's or trainer's opinion, the horse had been able to return to a lower, equivalent, or higher level of performance after discharge from the hospital. If the owner or trainer did not consider the horse to have returned successfully to its previous level of performance, it was established whether this was attributable directly to the occurrence of the condition treated with A-RLP or whether other factors had contributed to the eventual outcome. Therefore, at long-term follow-up, horses were categorized according to whether they had returned to previous use (at the same or higher level or at a lower level owing to the primary complaint, an unrelated lesion, or an unknown reason), had retired without attempt to return to previous use, had been euthanized (because of the primary complaint or for an unrelated reason), or were still convalescing. For some horses, long-term follow-up information was not available. 
Statistical analysis-Descriptive statistical analysis of data in different groups was performed. Outcomes that were evaluated included long-term outcome (alive vs euthanized for the primary complaint at long-term follow-up) and return to previous athletic use (same or higher level vs lower level because of the primary complaint). For each outcome, univariate exact logistic regression analysis was used to test the variables of interest to determine whether they were significant predictors of the outcomes alive versus euthanized for the primary complaint and returned to previous use at the same or higher level or at a lower level because of the primary complaint. Multivariate stepwise logistic regression analyses were then performed to fit prediction models for the outcomes with an entry value of $P \leq 0.4$ for inclusion in the model. Interactions among significant factors in the multivariate stepwise logistic model were analyzed. Values of $P \leq 0.05$ were considered significant. Odds ratios and $95 \%$ CIs were calculated for significant variables. Power for the multivariate stepwise analyses was calculated on the basis of the $\chi^{2}$ score and degrees of freedom upon termination of the model building. Values are expressed as median and range. All analyses were performed with standard software. ${ }^{a}$

\section{Results}

A total of 174 horses (36 sexually intact males, 54 castrated males, and 84 females) were included in the study. There were 56 Thoroughbreds, 53 Standardbreds, 23 warmbloods, 18 American Quarter Horses, 16 mixed-breed horses, 3 draft horses, 3 Arabians, and 2 ponies. The median (range) age at the time of admission was 4 years ( 0.003 to 20 years). Athletic use at the time of admission was recorded in 150 (86.21\%) cases and included racing $(n=60)$, performance (showjumping, dressage, or western showing; 32), pleasure riding (13), breeding (8), and other purposes (2). There were 5 yearlings or young horses that had not started training and 30 foals (age $\leq 6$ months). There were 96 horses with septic synovitis (group 1; 74 septic joints, 16 septic sheaths, and 6 septic bursae), 50 with lacerations (group 2; 27 fresh, minimally contaminated intrasynovial lacerations and 23 extrasynovial lacerations), and 28 with other conditions (group 3; osteomyelitis [4], fractures [2], prophylactic use of A-RLP [11], surgical site infections [5], and other conditions [6]). Median (range) duration prior to admission to the hospital was 4 days ( 0 to 60 days; group 1 ), 0 days ( 0 to 25 days; group 2), and 5 days (0 to 180 days; group 3 ).

The A-RLPs were performed under general anesthesia ( $\mathrm{n}=9$ [5\%]) or standing sedation (165 [95\%]). In 97 (55.74\%) horses, treatment with A-RLP was initiated the day of admission, and perfusions were repeated on a daily basis. The median (range) number of A-RLPs performed per horse was 5 (1 to 19), median (range) volume of the perfusate administered was $50 \mathrm{~mL}$ (12 to $62 \mathrm{~mL}$ ), and median (range) duration of application of tourniquet was 20 minutes (15 to 45 minutes). Addition of local anesthetic to the perfusate was recorded in 91 (52.2\%) cases; $2 \%$ lidocaine was used in most horses at a median (range) dose of $10 \mathrm{~mL} /$ perfusion (1 to 45 $\mathrm{mL}$ /perfusion). The use of a perineural block proximal to the tourniquet was recorded in 7 animals. Intravenous RLP was used in 155 (89.1\%) cases, and intraosseous RLP was used in 27 (15.51\%); in 8 (4.6\%) cases, both IV and intraosseous RLP were used. The cephalic and saphenous veins were used most often for IV RLP; the palmar or plantar digital vein was less frequently used. The dorsal metatarsal artery was used in 1 case. The medullary cavity of the third metacarpal or metatarsal bone was most commonly used for intraosseous RLP; the medullary cavity of the radius was injected in 1 instance. The type of tourniquet and the device used for injection were not recorded consistently. However, at our institution, a 12-cm-wide elastic tourniquet (Esmarch bandage) is typically placed proximal to the area to be perfused. Intravenous RLP is commonly performed with a 22-gauge butterfly needle, and custommade cannulated 4.5-mm cortical screws with a welded Luer-lock adaptor are typically used for the intraosseous route. At the end of the IV injection of the perfusate, the butterfly catheter is removed, and pressure is applied with gauze and several rounds of tape, which is removed after tourniquet release. After completing the intraosseous RLP, a sterile injection port is applied to the Luer-lock adaptor, and the limb is kept bandaged.

No major complications for the A-RLP procedure were reported. Vein-related complications (hematoma, phlebitis, or thrombosis) were recorded in 19 of 155 (12.26\% of horses undergoing IV RLP). Screw-related complications (discharge around the screw, difficult injection, screw loosening, and screw breakage) were recorded in 9 of 27 (33\%) horses undergoing intraosseous RLP. In the patient where the dorsal metatarsal artery was used, no complications were recorded. Median (range) hospitalization time was 11 days (2 to 74 days; group 1), 10 days (1 to 98 days; group 2), and 7 days (2 to 97 days; group 3). Long-term follow-up information was available for 132 (75.86\%) horses, with a median (range) time of 3.25 years ( 0.17 to 12 years) after discharge from hospital. From these 132 horses, $85(64.4 \%)$ had returned to their previous use and were included in the multivariate stepwise analysis for return to previous athletic use at the same or higher level or at a lower level because of the primary complaint.

Synovial infections (group 1)-There were 96 patients with synovial infections. The origin of infection was hematogenous in 18 (18.75\%) cases, iatrogenic (after intrasynovial injection or surgery) in 30 (31.25\%), traumatic (wound) in $44(45.83 \%)$, and unknown in 4 (4.16\%). In 26 (27.1\%) cases, only the forelimbs were affected, and in $64(66.7 \%)$ cases, only the hind limbs; in $6(6.2 \%)$ cases, both forelimbs and hind limbs were involved. In 78 (81.25\%) cases, only 1 synovial structure was affected, and in 18 (18.75\%), multiple synovial structures were affected. Seventy-four (77.88\%) infections were affecting joints, 16 (16.7\%) sheaths, and 6 (6.25\%) bursae. Synovial structures affected were tarsocrural joint $(n=38)$, metacarpophalangeal or metatarsophalangeal (fetlock) joint (24), distal interphalangeal joint (9), intercarpal joint (8), radiocarpal joint (6), proximal interphalangeal joint (4), stifle joint (3), tarsometatarsal joint (3), distal intertarsal joint (2), digital flexor tendon sheath (14), tarsal sheath (2), calcaneal bursa (5), navicular bursa (3), and digital extensor tendon sheath (1). 
Concurrent conditions were present in 34 (35.4\%) horses and included osteomyelitis $(\mathrm{n}=27$ [28.1\%]), tendon involvement (8 $[8.3 \%])$, and ligament involvement (2 [2.1\%]). Cases with osteomyelitis included 22 horses with septic arthritis, 2 with septic tenosynovitis, and 3 with septic bursitis. Of the 22 horses with septic arthritis and osteomyelitis, 12 cases were hematogenous, 7 cases were traumatic, 2 cases were iatrogenic, and 1 case was of unknown origin. Duration of the condition prior to admission to the hospital was distributed as $\leq 24$ hours $(\mathrm{n}=17$ cases $[17.7 \%]), 1$ to 7 days (45 [46.8\%]), $>7$ days (30 [31.3\%]), and unknown (4 [4.2\%]). Treatments prior to admission to the hospital included administration of antimicrobials systemically $(\mathrm{n}=71$ [74\%]) and intrasynovially (15 [15.6\%]).

In-hospital additional treatments included lavage (93 [96.9\%] cases, from which arthroscopy was used in 35 [36.5\%]), ISCL with isotonic fluids (14 [14.6\%] cases; 7 of these were septic tenosynovitis), systemic antimicrobials (95 [99\%] cases), and intrasynovial antimicrobials (60 [62.5\%] cases). Median duration of ISCL with isotonic fluids was 2 days (range, 1 to 7 days).

Bacterial culture and susceptibility testing were conducted in 81 (84.37\%) cases, of which positive bacterial growth was found in 48 (59.26\%). The most common systemic antimicrobial treatment used was the combination of penicillin and gentamicin initially $(\mathrm{n}=$ 67 [69.79\%]), followed by trimethoprim-sulfamide as a long-term treatment (26 [27.08\%]). Other less frequently administered systemic antimicrobials included ceftiofur, metronidazole, and enrofloxacin. In most foals, amikacin was used systemically instead of gentamicin, and the combination of clarithromycin and rifampin was also used in some cases. Amikacin was the antimicrobial most frequently administered into the synovial cavity ( $n=55$ [57.3\%]; $91.6 \%$ of horses receiving intrasynovial antimicrobials); other antimicrobials used included gentamicin, penicillin, meropenem, cefotaxime, and vancomycin.

Regional limb perfusion with antimicrobials was initiated the day of hospitalization in 46 (47.9\%) horses and the following day in 27 (28.1\%). Amikacin at a dose of 0.5 to $1 \mathrm{~g}$ /perfusion (range, $250 \mathrm{mg}$ to $4 \mathrm{~g}$ ) was most commonly used for A-RLP ( $n=78$ [81.25\%]). Gentamicin (300 $\mathrm{mg}$ [3 g/perfusion]) and ceftiofur (200 mg [ 1 g/perfusion]) were the next most commonly used antimicrobials. Other antimicrobials included timentin, cephazolin, cefotaxime, penicillin, cefoxitin, imipenem, meropenem, and vancomycin. Antimicrobials were combined in 17 cases: amikacin, timentin, and clavulanic acid $(n=3)$; gentamicin-amikacin and penicillin (9); cefazolin and amikacin (3); amikacin and cefoxitin (1); and ceftiofur and cephazolin (1).

At the time of discharge from the hospital (shortterm outcome), 69 (71.9\%) horses were alive, 22 (22.9\%) had been euthanized for reasons related to the primary complaint, and $5(5 \%)$ had been euthanized for other reasons. At long-term outcome, follow-up was available for 73 of 96 (76.04\%) horses, and from these, $39(53.43 \%)$ cases were alive, 26 (35.61\%) had been euthanized for the primary reason, and 8 (10.95\%) had been euthanized for other reasons. Sex was a significant $(P=0.03)$ factor for long-term survival. Mares (22 survived and 11 euthanized) were more likely to survive than geldings ( 10 survived and 11 euthanized; OR, 9.814; $95 \% \mathrm{CI}, 1.798$ to 53.559 ). Intact males (7 survived and 4 euthanized) were more likely to survive than geldings (OR, 5.331; 95\% CI, 0.619 to 45.9). Higher long-term survival rates (OR, 11.897; 95\% CI, 2.017 to 70.181 ) were associated with horses that received systemic administration of antimicrobials prior to admission to the hospital (25 survived and 15 euthanized), compared with those that did not receive antimicrobials (6 survived and 11 euthanized; $P=0.006$ ). Horses with a single synovial structure involved (33 survived and 17 euthanized) were more likely to survive long term (OR, 6.205; 95\% CI, 1.168 to $32.952 ; P=0.032$ ) than horses with multiple synovial structures involved (6 survived and 9 euthanized). Of the horses for which long-term follow-up was available that had returned to their previous use $(\mathrm{n}=35), 28(80 \%)$ were performing at the same or higher level, 6 (17.14\%) were performing at a lower level because of the primary complaint, and $1(2.86 \%)$ was performing at a lower level for other reasons. The probability to return to performance at a level equal to or higher than before the injury was higher for horses in which the hind limb was involved (23 higher vs 3 lower), compared with those in which the forelimb was involved ( 5 higher vs 4 lower; OR, 16.44; 95\% CI, 1.71 to $110.23 ; P=0.028)$. Horses that were not treated with ISCL with isotonic fluids were more likely to return to the same or higher level (27 higher vs 4 lower), compared with those in which ISCL with isotonic fluids was not used (1 higher vs 3 lower; OR, 43.99; 95\% CI, 1.929 to $>999.999 ; P=0.018)$. The following variables did not have a significant effect on survival or return to performance: duration of clinical signs before hospital admission, etiology of sepsis, presence of osteomyelitis, number of A-RLPs performed, use of intrasynovial antimicrobials, and use of arthroscopic or tenoscopic versus through-and-through lavage.

Lacerations (group 2)-There were 50 horses with lacerations. The front limb was affected in 22 (44\%) and the hind limb in 28 (56\%). Of the 27 acute, minimally contaminated lacerations with synovial involvement but without established synovial infection, 25 (92.6\%) had only 1 synovial structure involved, and 2 (7.4\%) had 2 synovial structures involved. Articulations were affected in 16 (59.25\%) cases and tendon sheaths in 10 (37.04\%), and 1 case involved a bursa (3.71\%). Synovial structures affected were fetlock joint $(n=8)$, tarsocrural joint (4), radiocarpal joint (1), proximal intercarpal joint (1), digital flexor tendon sheath (10), digital extensor tendon sheath (1), and calcaneal bursa (1).

Concurrent conditions were present in 22 (44\%) cases and included bone $(n=5[10 \%])$, tendon (15 [30\%]), or ligament involvement (2 [4\%]). Duration of the primary condition prior to admission to the hospital was distributed as follows: $\leq 24$ hours ( $\mathrm{n}=$ $41[82 \%]), 1$ to 7 days $(6[12 \%]),>7$ days $(2[4 \%])$, and unknown (1 [2\%]). Duration of the fresh, minimally contaminated intrasynovial lacerations $(n=27)$ was distributed as follows: $\leq 24$ hours (25 [92.6\%]) or 1 to 7 days (2 [7.4\%]). These 2 cases had a duration of clinical signs just over 24 hours but were included 
in group 2 on the basis of cytologic analysis, negative culture, and absence of obvious signs of synovial contamination. Additional treatments prior to admission to the hospital included systemic antimicrobials $(n=23$ [46\%]) and intrasynovial antimicrobials (1 [2\%]).

In the hospital, all horses received antimicrobials systemically. Other in-hospital treatments used in horses with intrasynovial lacerations included synovial lavage ( $\mathrm{n}=26$ [52\%] horses, from which arthroscopy was used in $5[10 \%])$, intrasynovial antimicrobials (12 [24\%]), and ISCL with isotonic fluids (7 [14\%]). The systemic antimicrobial treatment most commonly used was the combination of penicillin and gentamicin initially ( $\mathrm{n}$ $=37[74 \%]$ ), followed by trimethoprim-sulfamide as a long-term treatment (22 [44\%]). Other less frequently administered antimicrobials included ceftiofur and penicillin alone. Intrasynovial antimicrobials included ami$\operatorname{kacin}(\mathrm{n}=9[18 \%])$ and gentamicin $(3[6 \%])$

Regional limb perfusion with antimicrobials was initiated the day of hospitalization in 15 (53.57\%) horses and the following day in 9 (32.14\%). Amikacin at a dose of 0.5 to $1 \mathrm{~g} /$ perfusion (range, 0.150 to $3 \mathrm{~g} /$ perfusion) was most commonly used for RLP ( $n=42$ [84\%]). Gentamicin (1 g/perfusion), cefazolin (1 to 2 g/perfusion), and penicillin (both $2 \times 10^{6} \mathrm{U} /$ perfusion) were used occasionally. The median duration of the intrasynovial isotonic fluid CRI was 3 days (range, 1 to 5 days).

At the time of discharge from hospital (shortterm outcome), 47 (94\%) horses were alive, 2 (4\%) horses had been euthanized for reasons related to the primary complaint, and 1 (2\%) horse had been euthanized for other reasons. For long-term outcome, follow-up was available for 37 of 50 (74\%) horses, and of these, 34 (91.9\%) were alive, 2 (5.4\%) had been euthanized for the primary complaint, and 1 (2.7\%) had been euthanized for other reasons. Because of the small number of patients that were euthanized in this group, the degrees of freedom to test all the factors in a stepwise model were not available; therefore, logistic regression analysis was not performed for outcome survival.

Of the horses with long-term follow-up information available and that had returned to their previous use ( $\mathrm{n}=33), 24(72.72 \%)$ were performing at the same or a higher level, 8 (24.24\%) were performing at a lower level because of the primary complaint, and $1(3.03 \%)$ was performing at a lower level for undetermined reasons. Tendon involvement decreased the probability of return to previous use at the same or higher level $(P=0.020)$. Horses with lacerations not involving a tendon (18 vs 2 ) were more likely to return to performance at a level equal to or higher than before the injury than were horses with lacerations involving a tendon (6 higher vs 6 lower; OR, 9; 95\% CI, 1.418 to 57.117 ). The following variables did not have a significant effect on return to performance: duration of clinical signs before hospital admission, synovial involvement, type of synovial structure affected, systemic administration of antimicrobials prior to admission to the hospital, use of intrasynovial antimicrobials, number of A-RLPs performed, and use of tenoscopic vs through-and-through lavage.
Other conditions (group 3)-The 28 cases in group 3 were divided into surgical-site infections $(\mathrm{n}=5[17.86 \%])$, osteomyelitis (4 [14.29\%]), open fracture (2 [7.14\%]), prophylactic use of A-RLP (11 [39.29\%]), periarticular cellulitis (4 [14.29\%]), quittor (1 [3.57\%]), and bone sequestration (1 [3.57\%]). The forelimb was affected in 15 horses $(53.57 \%)$ and the hind limb in 13 (46.43\%).

Duration of the condition prior to admission to the hospital was distributed as follows: $\leq 24$ hours $(\mathrm{n}=7[25 \%]), 1$ to 7 days $(9[32.14 \%]),>7$ days (10 [35.72\%]), or unknown (2 [7.14\%]). In 8 horses (28.57\%), 1 synovial structure was involved. Systemic antimicrobials were administered in 24 (85.71\%) cases, and intrasynovial antimicrobials were used in 7 (25\%). Penicillin was the antimicrobial most commonly used systemically $(n=22$ [78.57\%]); other antimicrobials used included gentamicin, trimethoprim-sulfamide, and chloramphenicol.

Amikacin (125 mg to $3 \mathrm{~g} /$ perfusion) was used in A-RLP in all cases. Amikacin was combined with penicillin (2 cases) and ticarcillin and clavulanic acid (1 case).

At the time of hospital discharge, 26 (92.86\%) horses were alive, 1 (3.57\%) horse was euthanized for the primary complaint, and 1 (3.57\%) horse was euthanized for unrelated reasons. Twenty-two (78.57\%) horses were available for long-term outcome: 18 (81.82\%) were alive, 3 (13.64\%) were euthanized for reasons related to the primary complaint, and 1 (4.54\%) was euthanized for unrelated reasons. At that time, 17 horses had returned to their previous athletic use: 11 at the same or higher level (64.71\%) and 6 at a lower level (1 horse [5.88\%] for reasons related to the primary complaint, 4 [23.53\%] for unrelated reasons, and 1 [5.88\%] for undetermined reasons). No statistical analyses were performed in this group because of the diverse conditions included.

Power calculations-Power was calculated for the multivariate stepwise analysis for each of the groups. Results for power values were as follows for group 1 : alive versus dead, 93.67\%; return to previous use at the same or higher level versus at a lower level, $83.89 \%$. Results for power values were as follows for group 2: return to previous use at the same or higher level versus at a lower level, $71.57 \%$.

\section{Discussion}

The results of the present study confirmed the suggestions made in previous experimental investigations $^{6,12,13}$ and anecdotal reports $s^{1,5,14}$ that the use of A-RLP in horses is feasible and clinically safe. The technique can be easily performed with horses under standing sedation or general anesthesia. The IV route presented a lower incidence of technical complications than intraosseous injection; therefore, we suggest IV RLP would be preferable when venous access is available. The intra-arterial route was used in 1 case without complications; however, the arterial route was used only for the last perfusion on that horse because venous thrombosis occurred after 6 IV RLPs via the saphenous vein. The horse had a severe laceration to the right metatarsophalangeal (fetlock) joint, and the plan- 
tar digital veins were not available. The arterial route has been used for perfusion of the distal portion of the limb with miconazol in a mare with chronic pythiosis ${ }^{16}$; however, drug induced toxic effects on the endothelium are much more frequent and severe in arteries than in veins, ${ }^{17}$ and therefore, IV and intraosseous routes are preferred over intra-arterial RLP.

The IV route was most commonly used to perform A-RLP in the present study, likely indicating the readily accessible IV route versus the more invasive intraosseous RLP technique. Repeated daily venipuncture with a small-gauge needle was not associated with serious adverse effects, and topical anti-inflammatory drugs ${ }^{18}$ or intraperfusate dimethylsulfoxide ${ }^{1}$ were not used. Placement of indwelling IV catheters for A-RLP has been reported clinically ${ }^{1}$ and recently evaluated. ${ }^{19}$ Maintenance of indwelling catheters in limb veins of healthy horses for 5 to 7 days was associated with thrombosis in $15.78 \%$ of the veins, ${ }^{19}$ which is slightly higher than the complication rate after IV RLP in the present study (12.26\%).

The complication rate was higher for intraosseous RLP than IV RLP but included only minor complications related to the screw and the difficulty of injecting into the medullary cavity as previously described, ${ }^{10,20}$ and osteonecrosis of the injected bone $e^{3}$ did not occur. For repeated intraosseous RLP, the cannulated screw was maintained in place under a bandage and without routine administration of heparinized saline (0.9\% $\mathrm{NaCl}$ ) solution.

Signs of sepsis after administration of A-RLP have been reported in foals ${ }^{5}$ and humans ${ }^{21}$ but were not observed in this study. All the foals were receiving systemic antimicrobials at the time of the A-RLP, which could have decreased the likelihood of sepsis. ${ }^{21}$

Local anesthetics were included in the perfusate in some patients in the present study, both with IV and intraosseous routes. This practice may increase the comfort level of horses receiving intraosseous RLP $^{22}$ and might also increase the efficacy of the perfusion by reducing the movement of the patient. Perineural anesthesia proximal to the tourniquet was used in a limited number of cases, but in the authors' opinion, patients undergoing RLP with perineural anesthesia stand more still than those without perineural anesthesia.

In groups 1 and 2 in the present study, a series of 5 RLPs performed on consecutive days was typically administered regardless of the antimicrobial being used, but in severe infections and refractory cases, A-RLP was performed for longer periods or another series of 5 A-RLP was repeated. Repeated cytologic analysis of synovial fluid collected from the infected synovial structures is typically used by the authors as a guide to determine whether treatment should be continued. When A-RLP was used prophylactically (11 of the 28 cases in group 3), A-RLP was administered only once or twice. Amikacin was the antimicrobial most commonly used for RLP, probably because of its highest activity against pathogens isolated from equine orthopedic infections. ${ }^{23,24}$ Bacterial culture and antimicrobial susceptibility testing were performed on admission in most horses in group 1 , but A-RLP was typically started before the laboratory results became available. Antimicrobial doses varied widely, which is commonly observed in the literature. ${ }^{2,5,11,12,25}$ Regional limb perfusion with antimicrobials was repeated on a daily basis, although longer intervals (36 hours) have been indicated on the basis of studies ${ }^{22}$ performed on healthy horses. However, amikacin clearance was shortened in inflamed versus normal joints, ${ }^{26}$ and the septic acidic environment and accumulated cellular debris decrease the activity of some antimicrobials. ${ }^{27,28}$ Administration of antimicrobial combinations by RLP has been less frequently reported ${ }^{14}$ and was used in $9.8 \%$ of horses in this study. No specific complications were reported in those cases, but clinicians should be aware of the interactions among antimicrobials, ${ }^{29,30}$ which could affect the antimicrobial efficacy.

Fresh intrasynovial lacerations are commonly grouped together with septic processes in the literature $^{31,32}$; however, contamination differs from infection, and the response to treatment is therefore also different. It was our purpose to be as specific as possible when defining sepsis in a synovial structure and to evaluate septic synovitis and nonseptic contaminated intrasynovial lacerations separately. Previous studies ${ }^{15,24,33-39}$ evaluating septic synovitis in horses have found survival rates of $62 \%$ to $92 \%$ in adults and $42 \%$ to $85 \%$ in foals. In our study, $72 \%$ of horses with septic synovitis were discharged from the hospital, and $53.43 \%$ of the horses with follow-up information available were alive at long-term follow-up. These values are relatively low, compared with those reported; however, our definition of septic synovitis was more specific than in other studies, and $10.95 \%$ of horses with long-term follow-up information available had been euthanized for reasons unrelated to the septic condition. Foals and adults were evaluated together, but age was not a significant factor in the multivariate analysis. Our study population was purely referral, compared with other studies ${ }^{38,40}$ that also included primary cases. In fact, $74 \%$ of the patients in this study had received treatment by referring veterinarians before admission to the hospital, and $17 \%$ of cases had received intrasynovial antimicrobials. Therefore, a higher proportion of cases refractory to primary treatment might have been included, compared with other studies. ${ }^{38}$ Positive results of bacterial cultures have been associated with a lower survival rate. ${ }^{41}$ In group 1, 59.26\% of bacterial cultures were positive, which lies in the upper portion of the range of reported culture success rates (22\% to $74 \%)^{24,42-44}$ and might indicate high severity of disease. ${ }^{41}$ However, the effect of positive culture on rate of survival or return to performance was not investigated in our study.

In our study, systemic administration of antimicrobials before admission to the hospital was found to be a protective factor for survival in cases of septic synovitis. This finding supports the recommendation for early initiation of treatment and specifically the administration of antimicrobials. ${ }^{45}$ Duration of clinical signs prior to treatment has been negatively associated with prognosis in horses ${ }^{46,47}$ and has been reported as the most important prognostic indicator in humans. ${ }^{48}$ However, recent studies, ${ }^{31,37,39-41,49,50}$ including ours, have failed to find such an association. In the present study, median 
(range) duration of clinical signs before admission to the hospital in group 1 was 4 days (0 to 60 days), which is longer than in other studies, ${ }^{38,40}$ which have reported higher survival rates. Wereszka et $\mathrm{al}^{15}$ found that duration of clinical signs prior to admission to the hospital $<1$ day was associated with higher survival rates than clinical signs $>10$ days. Within the horses treated with A-RLP in this study, those with septic synovitis had lower survival rates (53.43\%) than horses with lacerations without infection (91.9\%); however, within each group, duration of the condition did not have a significant effect. Only $17.7 \%$ of the horses in group 1 were admitted to the hospital within 24 hours after the clinical signs were noticed, whereas $92.6 \%$ of the horses with intrasynovial lacerations in group 2 were admitted within 24 hours after the incident. Therefore, although duration prior to admission was not significant in this study, we believe that early treatment of injuries with synovial involvement is important for a successful outcome.

Geldings were less likely to survive, compared with mares and sexually intact males, in the present study. We speculate that this finding might be caused by greater economic constraints applied on geldings, compared with sexually intact males or mares that can be destined to breeding. The number of synovial structures affected was also a significant factor for survival; patients with only 1 synovial structure affected had a higher survival rate than patients with $>1$ synovial structure involved. This has been reported and is especially relevant in young foals. ${ }^{4,15,34,38,51}$ In this study, only 7 foals with multiple joints affected were included, and of those, only 3 survived. Concurrent osteomyelitis has also been reported to negatively influence prognosis $^{4,36,39,52}$; however, that was not observed in the present study. The high antimicrobial concentrations achieved in bone tissue after A-RLP ${ }^{22,53}$ might result in better outcome for these horses. In group 1, 5 foals with septic arthritis and osteomyelitis (1 with iatrogenic infection after Salter Harris II fracture repair and 4 with hematogenous septic arthritis and physitis) survived after treatment with systemic antimicrobials and A-RLP and without debridement of the osteomyelitic lesion. In group 3, 3 foals with septic physitis were included; all 3 survived, and 2 entered racing competition.

Survival or return to performance was not affected by the anatomic structure involved (ie, joint vs sheath vs bursa). Schneider et $\mathrm{al}^{24}$ reported a higher survival rate for horses with septic tenosynovitis, compared with septic arthritis, and the authors suggested that the absence of articular cartilage and milder negative consequences in septic tenosynovitis might have been the reason. It was also speculated that septic invasion of the subchondral bone could result in a persistent focus of infection difficult to resolve. Regional limb perfusion with antimicrobials might be more effective in preventing or treating infection in the subchondral bone, although gentamicin concentrations in subchondral bone after A-RLP were not different from those achieved after intra-articular administration in healthy horses. ${ }^{54}$

Reported rates for return to previous level of athletic use in horses with septic synovitis and lacerations to synovial structures are $40 \%$ to $60 \% .^{15,24,36,38-40}$ In our study, $48 \%$ of horses with septic synovitis returned to the previous or intended athletic use, and from those horses, $80 \%$ were performing at the same or at a higher level than before. Differences in study populations and treatment modalities make comparisons among studies difficult. In a recent report, ${ }^{55}$ the use of continuous intrasynovial antimicrobial infusion provided 94\% survival of horses with chronic or complicated septic synovitis, which compares favorably with our results. Continuous intrasynovial antimicrobial infusion could therefore be superior to repeated A-RLP when chronic septic synovial conditions are being treated, although the number of cases was limited.

Horses with septic synovitis treated with ISCL with isotonic fluids had worse prognosis than horses not treated with ISCL with isotonic fluids. The use of ISCL with isotonic fluids was to provide continuous lavage rather than a continuous administration of antimicrobials into the synovial cavity. Intrasynovial implantation of indwelling orthopedic drains has been used clinical$1 y^{24,33,49}$ and has been associated with an increased risk of ascending infection. ${ }^{49}$ The ISCL with isotonic fluids was used in 15 horses in group 1, and 7 of the 13 horses with long-term follow-up information available had been euthanized for the primary complaint. However, ISCL with isotonic fluids was used in 7 cases in group 2 , and 5 of the 6 cases with long-term follow-up information available were alive. Therefore, although ISCL with isotonic fluids did not seem to be protective over other treatments for septic synovitis, this treatment modality might have been used in more severe cases of septic synovitis.

The authors are not aware of any report that septic synovitis affecting the forelimb carries a worse prognosis for return to performance than the hind limb. A possible explanation could be that a higher proportion of the horse's weight is carried by the front limbs, ${ }^{56}$ and therefore postseptic consequences are more severe and performance limiting than those conditions affecting the hind limbs.

Horses with lacerations had an excellent prognosis for long-term survival (long-term survival rate, 92\%) and good prognosis for return to athletic use (89.2\%), regardless of whether the laceration was intrasynovial or extrasynovial and the type of synovial structure affected (ie, joint vs sheath vs bursa). From those horses that returned to athletic use, $72.7 \%$ were performing at a level equal to or higher than before the injury. These findings compare positively with those of other studies that have found long-term survival rates between $71 \%$ and $80 \%{ }^{31,32}$ and rates of return to athletic use between $50 \%$ and $80 \% .^{15,31,32,37,50}$ In these studies, ARLP was used only in a small proportion of the cases, among other locoregional techniques for administration of antimicrobials. ${ }^{32}$ The fact that the synovial involvement of the laceration did not have a significant effect on the outcome indicates that fresh lacerations with contamination of synovial structures carry as good a prognosis as extrasynovial lacerations, provided that adequate treatment is administered and A-RLP is indicated in the treatment of these cases. Even though duration prior to admission did not have a significant effect on survival or return to performance, our findings indicate that adequate treatment should be performed 
before the infection becomes established, which suggests early treatment carries a better prognosis in these conditions. ${ }^{15,24,32,33}$ Tendon involvement was the only factor that had a significant effect on return of the horse to performance, which is in agreement with results of other studies. ${ }^{32}$ Differences in the prevalence of tendon involvement in study populations may also be the reason for differences in outcomes among studies. ${ }^{32}$

As reported, ${ }^{24,38}$ the synovial structure involved did not have a significant effect on survival or return to performance. The tarsocrural joint was most commonly affected in cases of septic arthritis followed by the fetlock joint, which corresponds with the literature. ${ }^{24,35,51}$ When referring to synovial sheaths, the digital flexor sheath was mostly affected both in septic cases and in lacerations, as reported. ${ }^{15,31,32,40}$

Regional limb perfusion with antimicrobials was used for a variety of conditions in the horses in group 3. The prophylactic use of A-RLP has not been reported in the equine veterinary literature, but its effectiveness is well described in humans. ${ }^{57}$ This practice provides high tissue concentrations of antimicrobials when surgically approached and might be beneficial when horses undergo long surgical procedures, such as repair of complex fractures or difficult arthrodesis, which are associated with a higher risk of infection. ${ }^{58}$

The inclusion criteria and the retrospective nature of this study preclude conclusions regarding effectiveness of the technique. Regional limb perfusion with antimicrobials is usually used as an adjunct to conventional treatment (systemic administration of antimicrobials, lavage, debridement, administration of anti-inflammatory drugs, and rest), which also limits the evaluation of the effectiveness of RLP as a sole treatment. Treatment strategies and management can differ largely among cases, and this fact precludes direct comparisons among clinical reports. However, our results are favorable for the use of A-RLP in the clinical setting of equine medicine. The relative effectiveness of A-RLP compared with other modalities of locoregional antimicrobial treatment is also unknown. Studies adopting a prospective, controlled design are required to be able to draw conclusions on the efficacy of specific treatment regimens.

a. SAS OnlineDoc, version 9.1.3, SAS Institute Inc, Cary, NC.

\section{References}

1. Cimetti LJ, Merriam J, D'Oench SN. How to perform intravenous regional limb perfusion using amikacin and DMSO, in Proceedings. 50th Annu Conv Am Assoc Equine Pract 2004;14-29.

2. Rubio-Martínez LM, Cruz AM. Antimicrobial regional limb perfusion in horses. IAm Vet Med Assoc 2006;228:706-712.

3. Parker RA, Bladon BM, McGovern K, et al. Osteomyelitis and osteonecrosis after intraosseous perfusion with gentamicin. Vet Surg 2010;39:644-648.

4. Neil KM, Axon JE, Begg AP, et al. Retrospective study of 108 foals with septic osteomyelitis. Aust Vet I 2010;88:4-12.

5. Santschi EM, Adams SB, Murphey ED. How to perform equine intravenous digital perfusion, in Proceedings. 44th Annu Conv Am Assoc Equine Pract 1998;198-201.

6. Butt TD, Bailey JV, Dowling PM, et al. Comparison of 2 techniques for regional antibiotic delivery to the equine forelimb: intraosseous perfusion vs. intravenous perfusion. Can Vet I 2001;42:617-622.
7. Rubio-Martínez LM, López-Sanromán J, Cruz AM, et al. Evaluation of safety and pharmacokinetics of vancomycin after intraosseous regional limb perfusion and comparison of results with those obtained after intravenous regional limb perfusion in horses. Am I Vet Res 2006;67:1701-1707.

8. Rubio-Martínez LM, López-Sanromán J, Cruz AM, et al. Evaluation of safety and pharmacokinetics of vancomycin after intravenous regional limb perfusion in horses. Am I Vet Res 2005;66:2107-2113.

9. Pille F, De Baere S, Ceelen L, et al. Synovial fluid and plasma concentrations of ceftiofur after regional intravenous perfusion in the horse. Vet Surg 2005;34:610-617.

10. Whitehair KJ, Blevins WE, Fessler JF, et al. Regional perfusion of the equine carpus for antibiotic delivery. Vet Surg 1992;21:279-285.

11. Whitehair KJ, Adams SB, Parker JE, et al. Regional limb perfusion with antibiotics in three horses. Vet Surg 1992;21:286-292.

12. Whitehair KJ, Bowersock TL, Blevins WE, et al. Regional limb perfusion for antibiotic treatment of experimentally induced septic arthritis. Vet Surg 1992;21:367-373.

13. Scheuch BC, Van Hoogmoed LM, Wilson WD, et al. Comparison of intraosseous or intravenous infusion for delivery of amikacin sulfate to the tibiotarsal joint of horses. Am I Vet Res 2002;63:374-380.

14. Kettner NU, Parker JE, Watrous BJ. Intraosseous regional perfusion for treatment of septic physitis in a two-week-old foal. $\underline{\mathrm{Lm}}$ Vet Med Assoc 2003;222:346-350.

15. Wereszka MM, White NA II, Furr MO. Factors associated with outcome following treatment of horses with septic tenosynovitis: 51 cases (1986-2003). I Am Vet Med Assoc 2007;230:11951200

16. Worster AA, Lillich JD, Cox JH, et al. Pythiosis with bone lesions in a pregnant mare. LAm Vet Med Assoc 2000;216:17951798.

17. Gottlob R. Endothelschäden nach intravasalen Injektionen und Infusionen. Verh Dtsch Ges Pathol 1972:56:563-565.

18. Levine DG, Epstein KL, Neelis DA, et al. Effect of topical application of $1 \%$ diclofenac sodium liposomal cream on inflammation in healthy horses undergoing intravenous regional limb perfusion with amikacin sulfate. Am I Vet Res 2009;70:13231325.

19. Kelmer G, Catasus CT, Saxton AM, et al. Evaluation of indwelling intravenous catheters for the regional perfusion of the limbs of horses. Vet Rec 2009; 165:496-501.

20. Finsterbusch A, Argaman M, Sacks T. Bone and joint perfusion with antibiotics in the treatment of experimental staphylococcal infection in rabbits. L Bone Joint Surg Am 1970;52:1424-1432.

21. Finsterbush A, Weinberg H. Venous perfusion of the limb with antibiotics for osteomyelitis and other chronic infections. IBone Joint Surg Am 1972;54:1227-1234.

22. Mattson S, Boure L, Pearce S, et al. Intraosseous gentamicin perfusion of the distal metacarpus in standing horses. Vet Surg 2004:33:180-186.

23. Moore RM, Schneider RK, Kowalski J, et al. Antimicrobial susceptibility of bacterial isolates from 233 horses with musculoskeletal infection during 1979-1989. Equine Vet I 1992;24:450456.

24. Schneider RK, Bramlage LR, Moore RM, et al. A retrospective study of 192 horses affected with septic arthritis/tenosynovitis. Equine Vet I 1992;24:436-442.

25. Murphey ED, Santschi EM, Papich MG. Regional intravenous perfusion of the distal limb of horses with amikacin sulfate. I Vet Pharmacol Ther 1999;22:68-71.

26. Taintor J, Schumacher J, DeGraves F. Comparison of amikacin concentrations in normal and inflamed joints of horses following intra-articular administration. Equine Vet I 2006;38:189191.

27. Tack KJ, Sabath LD. Increased minimum inhibitory concentrations with anaerobiasis for tobramycin, gentamicin, and amikacin, compared to latamoxef, piperacillin, chloramphenicol, and clindamycin. Chemotherapy 1985;31:204-210.

28. Svensson E, Hanberger H, Nilsson LE. Pharmacodynamic effects of antibiotics and antibiotic combinations on growing and nongrowing Staphylococcus epidermidis cells. Antimicrob Agents Chemother 1997;41:107-111. 
29. Wallace SM, Chan LY. In vitro interaction of aminoglycosides with beta-lactam penicillins. Antimicrob Agents Chemother 1985;28:274-281.

30. Hershberger E, Aeschlimann JR, Moldovan T, et al. Evaluation of bactericidal activities of LY333328, vancomycin, teicoplanin, ampicillin-sulbactam, trovafloxacin, and RP59500 alone or in combination with rifampin or gentamicin against different strains of vancomycin-intermediate Staphylococcus aureus by time-kill curve methods. Antimicrob Agents Chemother 1999:43:717-721.

31. Frees KE, Lillich JD, Gaughan EM, et al. Tenoscopic-assisted treatment of open digital flexor tendon sheath injuries in horses: 20 cases (1992-2001). L Am Vet Med Assoc 2002;220:1823-1827.

32. Fraser BS, Bladon BM. Tenoscopic surgery for treatment of lacerations of the digital flexor tendon sheath. Equine Vet I 2004:36:528-531.

33. Schneider RK, Bramlage LR, Mecklenburg LM, et al. Open drainage, intra-articular and systemic antibiotics in the treatment of septic arthritis/tenosynovitis in horses. Equine Vet 1992;24:443-449.

34. Steel CM, Hunt AR, Adams PL, et al. Factors associated with prognosis for survival and athletic use in foals with septic arthritis: 93 cases (1987-1994). IAm Vet Med Assoc 1999;215:973-977.

35. Meijer MC, van Weeren PR, Rijkenhuizen AB. Clinical experiences of treating septic arthritis in the equine by repeated joint lavage: a series of 39 cases. I Vet Med A Physiol Pathol Clin Med 2000;47:351-365.

36. Post EM, Singer ER, Clegg PD, et al. Retrospective study of 24 cases of septic calcaneal bursitis in the horse. Equine Vet 2003;35:662-668.

37. Wright IM, Smith MR, Humphrey DJ, et al. Endoscopic surgery in the treatment of contaminated and infected synovial cavities. Equine Vet I 2003;35:613-619.

38. Smith LJ, Marr CM, Payne RJ, et al. What is the likelihood that Thoroughbred foals treated for septic arthritis will race? Equine Vet I 2004;36:452-456.

39. Stewart AA, Goodrich LR, Byron CR, et al. Antimicrobial delivery by intrasynovial catheterisation with systemic administration for equine synovial trauma and sepsis. Aust Vet 2010;88:115-123.

40. Smith LJ, Mellor DJ, Marr CM, et al. What is the likelihood that a horse treated for septic digital tenosynovitis will return to its previous level of athletic function? Equine Vet I 2006;38:337-341.

41. Taylor AH, Mair TS, Smith LJ, et al. Bacterial culture of septic synovial structures of horses: does a positive bacterial culture influence prognosis? Equine Vet I 2010;42:213-218.

42. Lavoie JP, Couture L, Higgins R, et al. Aerobic bacterial isolates in horses in a university hospital, 1986-1988. Can Vet 1991;32:292-294.
43. Honnas CM, Welch RD, Ford TS, et al. Septic arthritis of the distal interphalangeal joint in 12 horses. Vet Surg 1992;21:261-268.

44. Madison JB, Sommer M, Spencer PA. Relations among synovial membrane histopathologic findings, synovial fluid cytologic findings, and bacterial culture results in horses with suspected infectious arthritis: 64 cases (1979-1987). L Am Vet Med Assoc 1991;198:1655-1661.

45. Lugo J, Gaughan EM. Septic arthritis, tenosynovitis, and infections of hoof structures. Vet Clin North Am Equine Pract 2006;22:363-388.

46. Baxter GM. Retrospective study of lower limb wounds involving tendons, tendon sheaths or joints in horses, in Proceedings. 33rd Annu Conv Am Assoc Equine Pract 1987;715-728.

47. Gibson KT, Mcllwraith CW, Turner AS, et al. Open joint injuries in horses: 58 cases (1980-1986). L Am Vet Med Assoc 1989;194:398-404.

48. Bennett OM, Namnyak SS. Acute septic arthritis of the hip joint in infancy and childhood. Clin Orthop Relat Res 1992;123-132.

49. Honnas CM, Schumacher J, Cohen ND, et al. Septic tenosynovitis in horses: 25 cases (1983-1989). I Am Vet Med Assoc 1991;199:1616-1622.

50. Chan CC, Murphy H, Munroe GA. Treatment of chronic digital septic tenosynovitis in 12 horses by modified open annular ligament desmotomy and passive open drainage. Vet Rec 2000;147:388-393.

51. Martens RJ, Auer JA, Carter GK. Equine pediatrics: septic arthritis and osteomyelitis. IAm Vet Med Assoc 1986;188:582-585.

52. Paradis MR. Update on neonatal septicemia. Vet Clin North Am Equine Pract 1994;10:109-135.

53. Rubio-Martínez L, López-Sanromán J, Cruz AM, et al. Medullary plasma pharmacokinetics of vancomycin after intravenous and intraosseous perfusion of the proximal phalanx in horses. Vet Surg 2005;34:618-624.

54. Werner LA, Hardy J, Bertone AL. Bone gentamicin concentration after intra-articular injection or regional intravenous perfusion in the horse. Vet Surg 2003;32:559-565.

55. Lescun TB, Vasey JR, Ward MP, et al. Treatment with continuous intrasynovial antimicrobial infusion for septic synovitis in horses: 31 cases (2000-2003). L Am Vet Med Assoc 2006;228:19221929.

56. Ross MW. Conformation and lameness. In: Ross MW, Dyson SJ, eds. Diagnosis and management of lameness in the horse. St Louis: Saunders Elsevier, 2003;15-30.

57. Lazzarini L, Novelli A, Marzano N, et al. Regional and systemic prophylaxis with teicoplanin in total knee arthroplasty: a tissue penetration study. IArthroplasty 2003;18:342-346.

58. MacDonald DG, Morley PS, Bailey JV, et al. An examination of the occurrence of surgical wound infection following equine orthopaedic surgery (1981-1990). Equine Vet I 1994;26:323-326. 